\title{
Imaginarios urbanos sobre topofilia y topofobia: el caso de la ciudad de Chiguayante, Región del Biobío
}

\section{Urban imaginaries, topophilias and topophobias: The city of Chiguayante (Biobío region, Chile), a case study}

\section{Matías Medel Fernández* y Victor Montre Águila**}

Recibido: 21 de marzo de 2018

Aceptado: 19 de junio de 2018

\section{Resumen}

La modernidad y su asiento en obras de infraestructura, como elemento de memoria y construcción de capital social, constituye un modelo exquisito de soberanía y control integral del territorio, que se materializa también en una memoria social, a través de un paisaje cultural.

Se propone explorar en una dimensión cualitativa, la conformación de un paisaje cultural local y los cambios que se han producido en el territorio como matriz física, así como en la memoria de la comunidad de la ciudad de La Unión (Chile), a partir de la instalación de la central hidroeléctrica Llollelhue, más conocida como La Turbina. En una arquitectura como conjunto material, que considera escalas de control del recurso hídrico, y que es parte de un paisaje con atributos patrimoniales y de vocación productiva actual.

Esto se aborda metodológicamente desde la comprensión multiescalar de la dimensión del paisaje y de la memoria social vinculada, como elemento de la dimensión socio cultural. Los resultados preliminares apuntan a caracterizar esa matriz hipotética de dinámicas colaborativas entre paisaje y sociedad. Así_ este paisaje cultural desarrollado por La Turbina, se define como un sistema patrimonial que conjuga atributos que van más allá de su representación como bien inmueble y refieren una epistemología del territorio.

Palabras clave: arquitectura de la modernidad, memoria, paisaje cultural, patrimonio y sociedad.

\begin{abstract}
As an element of memory and social capital construction, Infrastructure projects constitutes an exquisite model of sovereignty and integral control of the territory that gets materialised in a social memory, through a cultural landscape.

This paper is set to explore, in a qualitative dimension, the development of a local and cultural landscape; the changes that has been produced in the territory as the physical matrix, as well as the memory of the community from the city of La Union (Chile), after the installation of the Llollelhue hydroelectric plant, also known as La Turbina [the turbine]. The architecture, the building that control the water supply, still remain as an important part of the city landscape, with heritage attributes and current profitable vocation.

The methodology uses a multiple-scales approach of landscape and social memory, linked as an element of the sociocultural dimension. The preliminary results point to characterize a matrix of the collaborative dynamics, between landscape and society. Thus, this cultural landscape developed by La Turbina, is defined as a heritage system that conjugates features that go far beyond the limits of a building and they refer an epistemology of territory.

Keywords: Collective Memory, Cultural landscape, Cultural Heritage and Society, architecture of modernity.
\end{abstract}

* Magíster @ en Procesos Urbanos Sostenibles, Universidad de Concepción, Concepción, Chile. Contacto: matiasmedel@udec.cl
** Magíster en Investigación Social y Desarrollo, Universidad Católica de la Santísima Concepción, Concepción, Chile. Contacto: victormontre@ udec.cl

Cómo citar: Medel, M. y Montre, V. (2018). Imaginarios urbanos sobre topofilia y topofobia: el caso de la ciudad de Chiguayante, Región del Biobío. Revista de Urbanismo, 38, 1-16. http://dx.doi.org/10.5354/0717-5051.2018.48702 


\section{Introducción}

La siguiente investigación, tiene como propósito, generar un abordaje de la emociones y sentimientos que presentan los vecinos de la ciudad de Chiguayante (Región del Biobío) por los lugares públicos y con la misma ciudad. Para el abordaje, la investigación se sostiene desde el estudio de los imaginarios urbanos, específicamente a través de la topofilia y topofobia. Es por lo anterior, que debemos entender a la ciudad, como algo más que una simple construcción material (Hiernaux, 2007) - siguiendo a las ciencias sociales - que se han enfocado, cada vez más al estudio del componente subjetivo de la ciudad, específicamente a los imaginarios urbanos.

Un abordaje, desde el estudio de los imaginarios urbanos, no busca dejar de lado el ámbito de construcción material de la ciudad, sino más bien, entender las relaciones entre lo material construido y el subjetivo urbano (Nogué, 2014; Lindón, 2007b), partiendo de la premisa que se influencian mutuamente. Es por lo anterior, como afirma Aliste (2011), los diferentes estudios realizados en el Área Metropolitana de Concepción (AMC) y sus diferentes comunas han buscado comprender la realidad territorial desde abordajes como el número de comunas, sus atributos administrativos, morfológicos, entre otros, dejando de lado enfoques como los imaginarios y la subjetividad urbana. Para complementar, es necesario aclarar, la importancia de la espacialidad de estos fenómenos, ya que son reflejos del comportamiento territorial de los urbanitas (Garrido, 2014).

La búsqueda de reconocer, los lugares topofílicos y topofóbicos, quiere decir el poner en relevancia a los lugares en la construcción de la ciudad, manifestándose los sentimientos de amor y rechazo y respondiendo la interrogante de por qué estos imaginarios son vivenciados en estos lugares y no en otros (Yory, 2003). Este reconocimiento de los lugares de amor y rechazo, entrega una herramienta para contrarrestar la dinámica actual de la ciudad, donde el fin se ve trastocado desde la lógica económica neoliberal (Lefebvre, 1978), generando una construcción de ciudad desde el enfoque de ciudad construida (Vera, 2012).

La comuna de Chiguayante pertenece a una de las 54 comunas que integran la Región del Biobío, inserta en la
Provincia de Concepción, además de componer el AMC (Rojas, Muñiz y García, 2009). La ciudad de Chiguayante tiene una particularidad que la hace destacar por sobre las demás, ya que es la única ciudad del $A M C$, que presenta una morfología urbana con una trama de ciudad lineal, generando la inexistencia de un centro claramente definido. Otro aspecto que destacar es la característica de ciudad dormitorio de Chiguayante, significando, que sus vecinas y vecinos desarrollan su vida cotidiana fuera de los limites urbanos. Por último, es igualmente interesante constatar, que la comuna es una de las más jóvenes del AMC, adquiriendo este estatus en el año 1996 (Municipalidad de Chiguayante, 2009).

La población de la comuna en el año 2012, según datos del INE (2012), fue de 128.162 habitantes, lo que corresponde al 6,4\% de la población regional. Al año 2020, según Guiñez y Gaete (2013), se estima que la población aumentará a 166.334 habitantes, lo que permite registrarse nuevamente como la tercera comuna con mayor población proyectada, con una equivalencia del $7,74 \%$ de la población total regional. La densidad poblacional en la comuna es de $1.854,7 \mathrm{hab} / \mathrm{km}^{2}$, posicionándola como la segunda con mayor densidad en la región. Por último, se destaca a Chiguayante como comuna netamente urbana, ya la población de la ciudad representa el 99,9\% del total de habitantes, según datos entregados en el Pladeco (2013).

Finalmente, es de suma importancia destacar los sectores de la economía en que se desarrollan los vecinos de Chiguayante, donde la población activa en el sector primario representa el 3,12\%, el sector secundario $14,18 \%$ y el terciario 82,70\% (CASEN, 2009 en Pladeco, 2013). De igual manera, se debe resaltar, que la comuna presenta el menor nivel de autocontención $(0,28)$ en el $A M C$, lo que Rojas et al., (2009) consideran como responsable el gran número de población que se desplaza a otras comunas para trabajar, lo que conlleva a Chiguayante a posicionarse como ciudad dormitorio para los trabajadores del AMC.

Según Aliste (2011), la expansión urbana del Gran Concepción fue generando carencias de servicios comunales, conllevando un bajo arraigo con el espacio comunal, especialmente hacia comunas periféricas como 
Chiguayante, quienes manifestaron efectos ambientales y sociales.

De esta manera, el objetivo de la investigación es describir y comparar los imaginarios urbanos sobre topofilia y topofobia en la ciudad de Chiguayante. Presentando la siguiente hipótesis, los habitantes de la ciudad de Chiguayante han construido espacios de topofilia escasos y con una relación superficial con el espacio-lugar.

A raíz de las reflexiones anteriores, resulta necesario preguntarnos ¿cuáles son los imaginarios sociales presentes en los habitantes de la ciudad de Chiguayante? ¿qué lugares vinculados a la topofilia y topofobia existen en la ciudad de Chiguayante? ¿qué factores inciden en la construcción de estos imaginarios sociales? y ¿es posible modificar el imaginario urbano de la ciudad de Chiguayante por medio de la intervención urbana?

El artículo se subdivide en 4 secciones, inicialmente se construye un marco teórico-empírico de los imaginarios urbanos, topofilia y topofobia. Luego, se presenta la metodología y los resultados de la investigación, siendo discutidos en función de las teorías presentadas anteriormente. Finalmente, se presentan los principales aprendizajes y proyecciones sobre el objeto de estudio en la ciudad de Chiguayante.

\section{Marco teórico-conceptual Imaginarios urbanos}

Las teorías discutidas y que presentaremos a continuación, son el imaginario urbano, que presenta un plano físico y social, la topofilia, asociada a la construcción de vínculos emocionales con el lugar, y la topofobia, entendida como un rechazo hacia el lugar.

Como es de esperar, la evolución del conocimiento y la percepción de las realidades en el mundo moderno, han vivido un cambio drástico durante los últimos años del S.XIX y continua en el S.XXI. Las ciencias sociales que se dedican al estudio y comprensión de lo urbano no han quedado al debe de este vuelco de paradigma, Zusman (2013) destaca la aparición de términos como: imaginación, imaginarios geográficos, geografías imaginarias o imaginativas que provienen del mundo de la Geografía Humana. Lindón (2007a; 2009) recalca, por otra parte, que las ciencias sociales a través de la antropología, geografía, y sociología han aumentado el interés por el cuerpo y las emociones, considerándolos como dos componentes esenciales de la vida.

La concepción de lo urbano tradicionalmente ha orientado su estudio a la obra física de la ciudad, pero los nuevos enfoques en el conocimiento han logrado diferenciar líneas de estudios sobre la misma. Como afirma Blanco y Gurevich (2006), Hiernaux (2007), Kuri Pineda (2013), la ciudad tiene dos corrientes de estudios, siendo la primera, la que se encarga de estudiar las morfologias materiales y los géneros de vidas resultantes de las mismas. Comprendiendo al espacio como algo natural,

(...) como contenedor o receptáculo de objetos, personas o acontecimientos y, en algunos casos, como mero escenario del quehacer humano. Así pues, el espacio aparece bajo esta mirada como algo dado por sentado y no como un proceso resultado de las relaciones sociales (Kuri Pineda, 2013, p.72).

Por lo tanto, la ciudad es reducida a lo material, con construcciones concretas, donde estos elementos responden un surgimiento temporal e historico, dejando plasmada la historia de la misma.

La segunda corriente de estudio, nace del interés de conocer el ambito subjetivo de la ciudad. Lindón proponé estudiar "el cuerpo y las emociones como una ventana para comprender la construcción social de la ciudad, de lo urbano y sus lugares, a través de los sujetos que lo habitan corporal y emocionalmente" (2009a, p.6). Esta segunda corriente, de la subjetividad urbana, ha sido solayada por la corriente de abordaje material de lo urbano durante largo tiempo (Kuri Pineda, 2013), pero hoy "viene a complementar, a dar un suplemento, a ocupar fracturas o huecos de lo que sí podemos conocer" (Canclini, 2007, p.90 en Zusman, 2012, p.52), a encontrar las tierras incognitas que estan en la mente y corazón de hombres y mujeres, haciendo referencia a Lindón (2007a), conocer el mundo de lo no sólido.

El nuevo enfoque, el de la subjetividad de la ciudad y lo urbano, no quiere dejar de lado la construcción material, si no más bien, poner en relevancia como la construcción de la ciudad en el ámbito material y social tiene consigo una carga subjetiva - "son porciones del territorio inbuidas de significados, de emociones y, por lo tanto, llenas de significados para los seres humanos" (Nogué y Romero, 2006 en Nogué, 2014, p.157) -, además 
como esta carga subjetiva tiene consecuencias en el ambiente construido. En esta misma perspectiva, Aliste (2011) hace referencia a que el estudio de los significados entregan interesantes señales sobre las dinámicas urbanas pasadas, recientes y futuras.

Como afirma Hiernaux (2007), la subjetividad en lo urbano tiene injerencia en la construcción, tanto del pensamiento, del actuar individual y el social, entendiéndolas como un "conjunto de simbolos, representaciones, ideas, teologías, tecnologías, entre otras cosas, que configuraron, configuran y configuraran las áreas urbanas" (Medel Fernández, 2016, p.22), en donde se entrega mayor preponderancia a "los procesos por sobre el determinismo marcado por las explicaciones basadas en causa y efecto" (Vera, 2012, p.78). Es decir, lo subjetivo y lo material tiene un relación de mutua influencia el uno con el otro.

Complementando lo anterior, durante los últimos siglos se han generado cambios de paradigmas significativos, que llevan a (re)pensar el como abordar la realidad urbana, donde se destaca la aparición de la fenomenología, cual presta una mayor atención y un manejo mas cuidadoso, sobre el comportamiento y los fenómenos que ocurren de estos, ligados a la imaginación y la subjetividad. Si bien, se ha posicionado esta forma de abordar la vida urbana y la construcción de la misma (imaginarios urbanos), hoy en día sigue existiendo corrientes científicas que obvian o no le dan la real preponderancia a esta tematica, a pesar como afirma Hiernaux (2007) cuando destaca la obra de Durand: "este autor desarrolla los argumentos necesarios como para que ya no sea posible negar la fuerza de los imaginarios, así como para avanzar en su reconocimiento como elemento central (aunque no único) para comprender el comportamiento humano" (Hiernaux, 2007:20).

Esta falta de abordaje, desde la perspectiva del imaginario urbano, desde la subjetividad misma de la ciudad y el comportamiento de sus urbanitas, se debe a que representa una mayor complejidad :

(...) la complejidad es un modo de ver el mundo que implica una perspectiva múltiple y relacional. Este modo no cartesiano significa más un desafío que un esquema acabado, ya que incita a pensar de manera compleja la acción cotidiana en cualquier campo social (Vera, 2012:73).
Finalmente, solo queda destacar, que el abordaje de la subjetividad en lo urbano ha llegado para posicionarse como un elemento central, pero no dejando de lado otros diferentes enfoques, si no más bien, generándose complementos que deben ser entendidos como un todo, como un sistema indisociable, que se complementan y tienen influencias el uno con el otro, cual invita a llevar a cabo un relectura de cómo entender la vida urbana.

\section{Espacialidad de lo subjetivo}

La relación entre espacio y subjetividad debe ser destacada, es por esto que se afirma, al igual que Aliste (2011; 2015; 2016), Bailly (1989), Harvey (1990), Hiernaux (2007), Lindón (2007a; 2007b; 2009), Pyszczek (2012), Vera (2012), Rizo (2012), Kuri Pineda (2013), Zusman (2013), Garrido (2014), Nogué (2014), Yory (2007), Gallardo Frías (2011), Uribe (2002), que el espacio es el lugar en donde se llevan a cabo los imaginarios, por lo tanto, eje vertebral de la vida social, "organizamos nuestra relación con el entorno y los demás sujetos con quienes interactuamos" (Rizo, 2006, p.5). La espacialidad de los imaginarios, como afirma Lindón (2009), tiene que ver con la concepción del sujeto como un ente espacial y es este quien carga de elementos subjetivos a lo que solo son espacios.

La aseveración de que lo espacial y los subjetivo presentan una relación de suma importancia, deja de lado la concepción de que el tiempo era el principal componente de lo espacial, "percepción paradigmatica que llevó a que durante el s.XIX y buena parte del XX el tiempo fuese dinámico y el espacio lo inerte" (Aliste $y$ Nuñez, 2015, p.87). Hiernaux ratifica esta concepción relacional entre el espacio y lo subjetivo, al proponer que "la espacialidad misma de las imágenes afirma, así, un lazo fundamental, quizás fundacional entre el espacio y el imaginario, que el tiempo no puede invocar, ya que la imagen es atemporal" (2007, p.21), en palabras simples, un imaginario transpasa la barreras temporales, permitiendo que estas sean guardadas y puedan manifestarse en otros instantes, generando interpretaciones diferentes del mismo imaginario, pero por el contrario, el espacio esta cargado de significancia en todo momento y además sufre mutuaciones continuas.

Por lo mismo, se destaca el rol escencial del espacio en la activación de los imaginarios. Siguiendo a Garrido 
(2014), los espacios son generadores de niveles de asociacion por las sensaciones que estos producen en los sujetos-sentimientos, ya que el urbanita está en una constante recepción de estímulos, que en palabra de Hiernaux - "conforman una suerte puesta bajo tensión permanente, para la percepción humana" (Hiernaux, 2007, p.22) - esto quiere decir, que el urbanita como sujeto esta en vigilia de estímulos (casi permanentes), por lo que está en una construcción incesante de imaginarios. Pero, es oportuno resaltar, que cuando se habla de que el urbanita esta en una recepción constante de estímulos, es en el espacio público donde se lleva a cabo esta dinámica, como subraya Vera (2012), la relación entre espacio e identidad se dan es espacios abiertos, dinámicos y activos, y esto jamás se logran en espacios cerrados que tienen por esencia el excluir, además que la ciudad es ciudad, en cuanto entra en juego lo público.

Esta nueva aceptación, de que el espacio tiene un componente clave que es la subjetividad, permite según Zusman (2013), generar una diferenciación entre los espacios para su abordaje, una diferenciación que toma en cuenta las formas de imaginar el medio, de apropiarse de él y actuar en este, como afirma Kuri Pineda (2013), las configuraciones sociales siempre se espacializan, reafirmando la necesidad de un abordaje espacial de las subjetividades.

La ciudad no puede ser abordada solo desde la costrucción física y las relaciones que se llevan a cabo en las misma, "el espacio social va de la mano con el espacio físico" (Kuri Pineda, 2013, p.73), es que se recalca el interés de la apropiación y costrucción desde el enfoque de los imaginarios urbanos. Como afirman Aliste y Núñez (2015) el sujeto deja de ser entendido como un expectador del lugar, pasa a ser un transformador de el a través de su interpretación y carácter sociocultural del mismo.

Vera (2012), denomina este tipo de concebir, construir y estudiar la ciudad como "ciudad construida", donde los imaginarios construyen las relaciones sociales y las diferentes infraestructuras, teniendo en cuenta los componentes de creatividad, valor e imaginación, permitirán abordar la realidad urbana, que en sí misma es compleja.

Es por lo anterior, que un abordaje meticuloso y correcto (uso de una metodología, que no quiere decir un abordaje rígido), permitirá al investigador y planificador de ciudad "participar con él (lector) de las impresiones que el lugar o la circunstancia, ha dejado en nosotros: hacerlo descender de su elevado puesto de mira y hacerlo ver con nuestros propios ojos y sentimientos" (Wright, 1997, p. 177 en Zusman, 2013, p.53), reafirmando el abordaje desde una concepción de ciudad construida.

Este entendimiento de ciudad construida, desde el estudio, debe lograr "establecer las relaciones entre la forma espacial, el significado simbólico y el comportamiento espacial" (Harvey, 1985, p.26 en Zusman, 2013, p.55), de esta forma generar un entendimiento acabado al momento de planificar, gestionar y/o construir ciudad.

Resumiendo, la importancia de dar el rol central a los imaginarios permite que, a través del entendimiento de estos, se logren "desarrollar procesos creativos $y$, particularmente sus implicancias en la transformación de la geografía material" (Zusman, 2013:59). Por lo tanto, no es descabellado pensar, que un imaginario puede llevar a tener implicancias tan importantes como el drenaje de un humedal, conservación de lugares esenciales para la ecología urbana, construcción de centros comerciales, derrumbamientos de lugares históricos, construcción de centros comunitarios, entre otras construcciones que se pueden llevar a cabo en la ciudad. En fin, como afirma Zusman (2013), el abordaje adecuado de la subjetividad (imaginarios) nos permite construir o no un mundo más habitable, entendiendo que la experiencia está anclada siempre a un espacio en donde se desarrolla.

\section{Topofilia}

Cuando se habla de topofilia, se debe comprender más allá de un simple concepto, de una forma de abordar y estudiar el comportamiento social, más bien, se debe entender como una acción, un sentimiento, una forma de actuar en el espacio, es un habitar cual nos permite interpretar y transformar el hábitat. El connotado geógrafo americano Tuan, define la topofilia como "el lazo afectivo entre las personas y el lugar o el ambiente circundante" (Tuan, 2007, p.13), pero que lazos afectivos y como se manifiestan estos son interrogantes que se pretenden responder. Por consiguiente, para comprender de forma más acabada el concepto de topofilia, es 
necesario hacer una pequeña revisión del origen de este enfoque imaginario-espacial.

La construcción de la palabra topofilia, responde a la composición de dos palabras, topos y philia. Se trata topos, siguiendo a Alguacil (2008), Foulkes (2002), Ortega et. Al, (2013) y Yory (2006; 2007; 2009a; 2009b), a la forma de estar, relacionarse o estar con las otras y los otros (comunidad), "cierta manera socio-espacial de comportarse y, por lo tanto, una actitud política (Yory, 2007, p.52), es decir, los modos de estar y comportarse (de forma ética) en un espacio determinado, cual es compartido, lo que tiene un impacto social $y$ consecuencias espaciales. Complementando, cuando se habla de topos, se alude a un espacio integrador y común, Yory (2006; 2007; 2009a; 2009b) lo define, como un lugar de comunidad, donde las y los miembros se sienten ligados el, consigo mismos, con el entorno y con las y los habitantes. Es por lo anterior, que topos, es dador y portador de imaginarios, generador de espacios construidos y social.

En conclusión, como topos es una construcción social, está en una recepción e interacción constante con los imaginarios,

(...) no se entenderá como una reunión de individuos previamente autónomos sino un conjunto de personas que se conciben a sí mismas en función de su pertenencia a la ciudad. No son individuos extremadamente relacionados con la ciudad, sino personas internamente relacionadas unas con otras y con la ciudad (Alguacil, 2008, p.51) .

Por consiguiente, no es un simple espacio receptáculo, más bien es el lugar de espacialidad de los imaginarios.

De lo anterior, se establece que topos, según Yory (2007), se entiende como un lugar de significación para los urbanitas, por lo que genera imprescindiblemente una philiación, entre los sujeto-sentimiento y el lugar, donde se comprometen en la construcción y apropiación del entorno. Complementando, Calvo Martínez (2003) define, la philiación como un sentimiento de amistad, lo que puede ser llevado al ámbito espacial, con el amor, cariño, cuidado, entre otros adjetivos (positivos), que tiene el sujeto-sentimiento con el lugar que habita.

La philiación, nos determina como "seres históricossociales y, por lo tanto, culturales, da cuerpo al propio sentido del lugar en el cual habitamos como un lugar cultural" (Yory, 2009:98). Con la philiación se humaniza los espacios, generando lugares, entregando sentido, que nos hace ser frente al y en el mundo.

Ya conociendo el origen etimológico de topofilia, se puede abordar de mejor forma la concepción de lugar y la carga de imaginarios de estos. Yory (2006) define la topofilia como una herramienta para diferenciar los espacios mesurables, de los vivenciados o vividos, ya que habitando cargamos de significancia los espacios que son solo mesurables sin la emoción entregada por los sujetosentimiento. La entrega de significancia, según Aliste (2011), Kuri Pineda (2013), Lindón (2004; 2009) y Yory (2007), se debe al actuar espacial del sujeto-cuerpo en conjunto con el sujeto-sentimiento, ya que además de ser espaciales, somos espaciantes a través de nuestra forma de actuar y de cargar imaginarios en los lugares. La carga de significancia de los espacios responde según Tuan (2007) a la recepción de estímulos que son percibidos por nuestros sentidos, con especial énfasis en la vista, como este autor afirma, pero sin dejar de lado que también los otros sentidos nos permiten enclavar significados en los lugares.

La capacidad de ser seres espaciantes, para Kuri Pineda (2009), hace existir un vínculo de mutua influencia entre el espacio y el sujeto-cuerpo y sujeto-sentimiento, afianzando aún más su conexión. A condición de lo anterior planteado, para lograr un abordaje más completo sobre topofilia y sus componentes espaciales, esta investigación se servirá de una diferenciación netamente investigativa, generando dos grandes categorías topofilia, simbólica-emocional y social-relacional, reiterando que dicha separación solo se realiza con fines investigativos, para generar una mejor comprensión del fenómeno espacial, ya que ambas se manifiestan de manera simultánea e indisociable la una de la otra.

\section{Topofobia y toponegligencia}

Sería faltar a la verdad el decir que los lugares solo se construyen de sentimientos positivos, sino que también estos generan y son portadores de sentimientos de que evocan una dimensión negativa, a estos sentimientos de desagrado por el lugar, se les llama topofobia. La topofobia es definida como, "contrapelo de lo que es la topofilia [...] implica el sentimiento de rechazo o desagrado hacia un lugar" (Kuri Pineda, 2013: 91). 
También es definida por Lindón (2009), como un grupo de sensaciones, cuales hacen que el sujeto-sentimiento genere sentimientos, los cuales provocan algún rechazo a un lugar específico.

Los lugares que son cargados por sentimientos negativos y, por lo tanto, cargados de topofobia pueden ser definidos como espacios peligrosos, donde se proyectan los miedos, la inseguridad y, por lo tanto, provocan rechazo. Los espacios topofóbicos, son lugares en donde permanecer se vuelve desagradable para los habitantes.

Lindón explica de forma clara las consecuencias de la topofobia:

(...) el sujeto-cuerpo se constituye en un activo constructor de distancias espacial y social con los otros y de alejamiento de un lugar. La lógica corporal es de tipo diastémica y topofóbica. El sujeto-cuerpo es el que, orientado por el rechazo, el desagrado y a veces el miedo, por el lugar y los otros que lo habitan, genera pasos y vivencias efímeras en los lugares. Para el sujeto-cuerpo el lugar sólo constituye un espacio atravesado (Lindón, 2009, p.16).

Este fenómeno, tiene una consecuencia política de actuar, una deformación ética en los habitantes, lo que es denominado por Yory (2001; 2003) como toponegligencia, entendiendo esta como una falta de compromiso y apego con cierto lugar de la ciudad, y como consecuencia no se experimenta una relación responsable y de pertenencia con el lugar. Lo que es más preocupante, es que este fenómeno avanza, como considera Lefebvre (1978) y Yory (1998), la dinámica de construcción de la ciudad trastocada, cual la búsqueda de réditos económicos ha dejado de lado la principal característica de las ciudades, que es generar espacios óptimos para la vida social y pública.

\section{Metodología}

La investigación posee un enfoque cualitativo, por medio del desarrollo de un diseño descriptivo, interpretativo y comparativo del objeto de estudio. El contexto de la investigación se vincula a la ciudad de Chiguayante, en Concepción, la cual fue subdividida en 6 áreas urbano-territoriales, en función de los siguientes criterios:

- Diferencias físicas en la trama urbana, relacionados con la continuidad.

- Barreras físicas, como calles y veredas en la ciudad de Chiguayante.

- Nivel socioeconómico basado en manzanas censales del INE-IDH. 


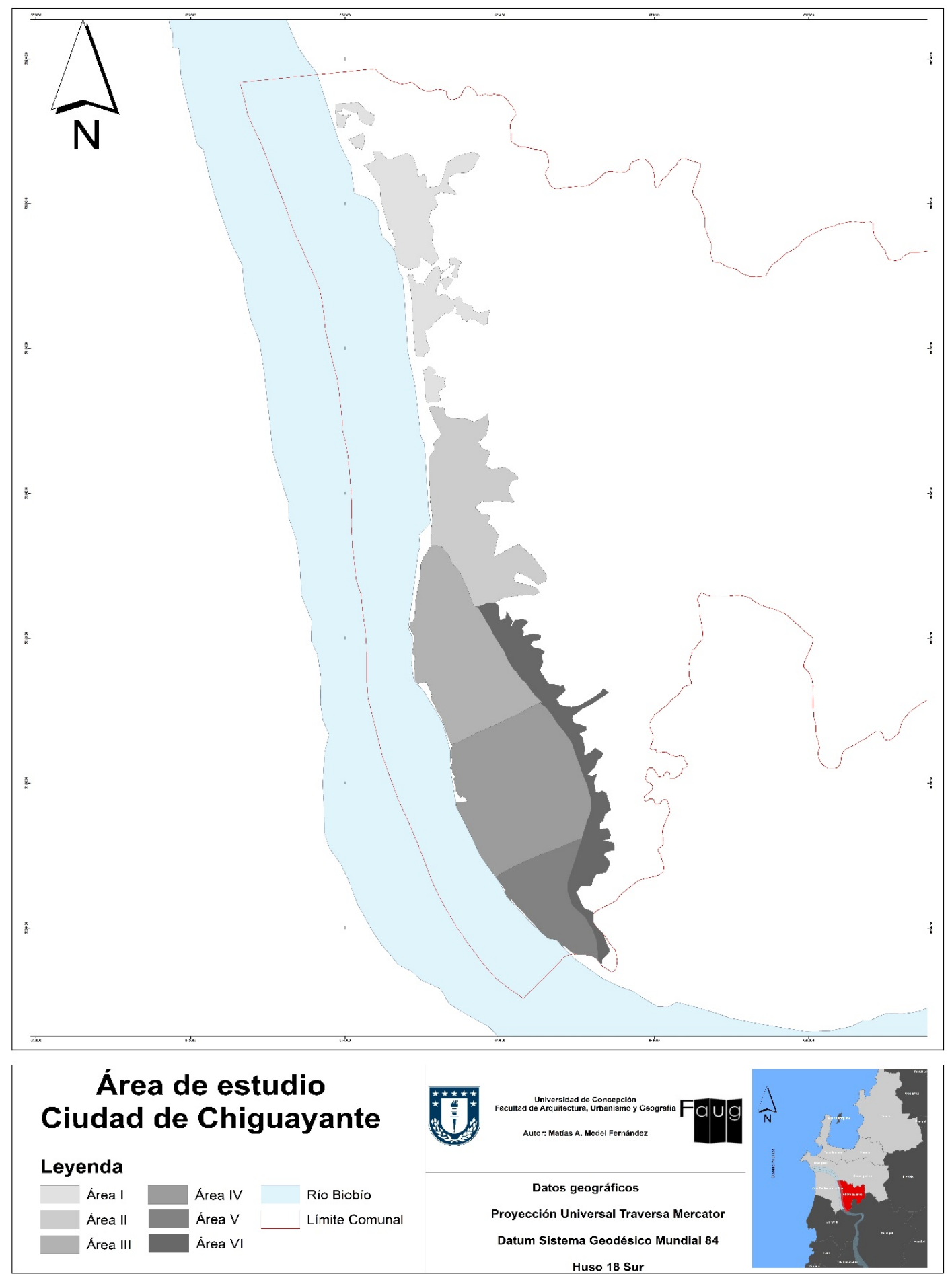

Figura 1. Áreas de estudio de la ciudad de Chiguayante Fuente: Elaboración de los autores. 
La muestra fueron 18 informantes claves, de las zonas territoriales de la ciudad de Chiguayante (ver figura 1), presentando los siguientes criterios de selección:

- Elección de 3 entrevistados por cada zona territorial.

- Informantes mayores de 18 años de edad.

- Tener residencia, no menor a 1 año, en la ciudad de Chiguayante.

La recolección de la información, se realizó durante los meses de septiembre a octubre del año 2016, a través de la técnica de entrevistas semiestructuradas, compuesta por 2 categorías y 10 reactivos. La primera categoría, se vincula a la calidad del espacio físico-público y la segunda es la calidad del escenario social. Cada informante clave firmo una carta de consentimiento al momento de ser entrevistando, aceptando su uso en esta investigación.

La información recopilada con la técnica de muestreo será tratada con los siguientes métodos, técnicas y software computacional. Las entrevistas fueron transcritas y se aplicaron 2 técnicas de análisis sobre la información recolectada. Inicialmente se realizó un análisis de frecuencia de códigos con un primer nivel de profundidad (Flick, 2004), a través del software de código libre QDA Miner Lite. Además, se desarrolló un análisis de mayor profundidad, a través de la teoría fundamentada (Strauss y Corbin, 1998), identificando categorías abiertas, axiales y selectivas (tres niveles de profundidad).

\section{Resultados y discusión}

La investigación a través de la codificación abierta, identificó 22 códigos, que presentan una alta relevancia para describir las relaciones físicas y sociales que existen en la comuna de Chiguayante. De esta manera, se construye una codificación selectiva, con una estructura que contempla temática, dimensión y código (ver cuadro 1). Luego de esto, se presentarán las reflexiones en dos líneas de discusión. La primera, se vincula con un tratamiento de la información desde una óptica teórica de los imaginarios urbanos; y una segunda línea construida desde la topofilia y la topofobia hacia los resultados.

Tabla 1. Codificación selectiva de las entrevistas

\begin{tabular}{|c|c|c|}
\hline Temática & Dimensión & Código \\
\hline \multirow[t]{11}{*}{ Espacio Físico } & \multirow[t]{8}{*}{ Espacios públicos } & Descripción de EP \\
\hline & & Déficit \\
\hline & & Uso \\
\hline & & Importancia \\
\hline & & Carencias \\
\hline & & Calidad \\
\hline & & Seguridad \\
\hline & & Accesibilidad \\
\hline & \multirow[t]{3}{*}{ Conocimiento sobre los EP } & Desconocimiento \\
\hline & & Vinculación con el Gran Concepción \\
\hline & & Chiguayante como dormitorio \\
\hline \multirow[t]{8}{*}{ Espacio Social } & \multirow[t]{3}{*}{ Uso del espacio } & Lugares reconocidos \\
\hline & & Espacios de conmemoración \\
\hline & & Vida social \\
\hline & \multirow[t]{4}{*}{ Organizaciones sociales } & Participación \\
\hline & & Desconocimiento \\
\hline & & Acciones \\
\hline & & Limitantes e interés \\
\hline & Oferta de servicios & Capacidad de oferta de Concepción \\
\hline
\end{tabular}

Fuente: Elaboración de los autores con base en las entrevistas de informantes claves 


\section{Espacio físico}

El espacio físico, conlleva una descripción basada en la opinión de los residentes de las diferentes zonas de Chiguayante, que identifican dos dimensiones. La primera dimensión son los espacios públicos (EP), desarrollando 6 códigos vinculados a un reconocimiento, al uso, su importancia, las carencias, la calidad y la accesibilidad de Ios EP; la segunda dimensión, busca profundizar en los conocimientos de los EP, describiendo el estado de desconocimiento sobre los EP en Chiguayante, una fuerte vinculación con la ciudad de Concepción y una noción de ciudad dormitorio.

La dimensión de los espacios públicos es descrita de la siguiente manera:

Descripción de los espacios públicos. Los informantes claves describen lugares que tengan importancia para los residentes de las diferentes áreas de la ciudad de Chiguayante.

Las plazas...la plaza de Chiguayante la que está en O’Higgins, bueno aquí todas las plazas de aquí (Lonco), las calles...la plaza de los Castaños (Entrevistado 2)

Uso de los EP. Los espacios públicos de la ciudad, tienen mayor o menor uso, debido a que los espacios son de menor o mayor espacio y seguridad en función del sector socioeconómico.

Déficit de los EP. En la mayoría de las áreas estudiados, existe una percepción de la insuficiencia en su cantidad, producto de las condiciones de una ciudad lineal.

Faltan muchos espacios públicos...no hay muchos si uno compara con otros lugares. Falta un parque igual...igual falta otro estadio o algo parecido, para hacer conciertos...porque acá no hay nada (Entrevistado 4)

Importancia a los EP. Hay lugares que son de mayor importancia para los residentes, especialmente las plazas de la ciudad, pero que no presentan un alto uso de ellos, al ser Chiguayante, una ciudad dormitorio.

Voy regularmente a la plaza de 8 Oriente...llevo a mi hijo a ver el árbol de pascua que ponen todos los años y también voy a las ferias costumbristas que se realizan de vez en cuando (Entrevistado 3).
Las carencias de los EP. Los espacios presentan fuertes carencias, en cuanto al acceso, las dimensiones, instalaciones y posición espacial de los lugares en la ciudad de Chiguayante.

La calidad de los EP. Los espacios utilizados no presentan altos niveles de calidad, su representación como áreas cafés, poco cuidado de las instalaciones, luminosidad, protección, cercanía a vías utilizadas por autos a alta velocidad, etc. inciden en una percepción negativa de los lugares de uso público.

La calidad es regular, porque a pesar de que hay espacios que son buenos, como las calles y los caminos, esas cosas, hay ciertas limitancias con algunos otros...los espacios de transporte están súper bien, pero los de recreación la verdad es que no (Entrevistado 16).

Las plazas, aunque me incluyo en eso, van a quemarla y hay gente que no le gusta o creen que son delincuentes. La plaza de Chiguayante, nica camino a esa hora relajado y en Los Castaños, tampoco caminaría relajado a esa hora (04:00). Leonera, ahí sí que no me sentiría tranquilo caminando a ninguna hora, pero lo digo sin conocer. Los sectores que están más cerca del río, como se ven siempre solos y cochinos (Entrevistado 1).

Seguridad de los EP. El tipo de plano urbano, las distancias, la percepción de un escaso cuerpo de carabineros conllevan a que los lugares son poco seguros, restringiendo su uso.

Son tranquilas (plazas de Lonco), nunca vas a ver patos malos haciendo nada, ni cabros malillas molestando a los demás. Los más seguros creo que son las plazas de aquí (Lonco)...y también debería ser la Plaza de Armas y la de los castaños, porque son importantes (Entrevistado 2).

Accesibilidad. La morfología urbana de Chiguayante no promueve un espacio planificado para la construcción de espacios públicos, la mayoría de los EP presentan características de estrechez y una escasa conexión con sus espacios circundantes. 
La dimensión de los conocimientos sobre los EP es desarrollada a través de:

Desconocimiento de los EP. La ciudad, se identifica, como una ciudad dormitorio, otorgando una alta relación con la ciudad de Concepción. Esta realidad, implica un desconocimiento de los espacios públicos existentes en la ciudad, ya que, la interacción interna es casi inexistente.

Pero jamás he pasado de la calle Esperanza, para allá esta Leonera, Los Bloques, dicen que es peluo, pero nunca me he aventurado por ahí (Entrevistado 3).

Vinculación con el Gran Concepción. A diferencia de la realidad anterior, los residentes se conectan fuertemente con el Gran Concepción por temas laborales, estudios, recreación, etc. identificando y describiendo fácilmente los EP de Concepción.

Chiguayante como dormitorio. Los residentes aceptan que su relación con su ciudad, es principalmente de uso para descansar, es decir, la ciudad se entiende como dormitorio, pero las funciones laborales, estudio, recreación son realizadas en el Gran Concepción, especialmente en Concepción.

Vengo acá casi a dormir no más...solo llego acá a descansar y me voy temprano en la mañana (Entrevistado 12).

Los imaginarios urbanos, entendiéndolos como un plano físico y social (Nogué, 2014; Hiernaux, 2007; Vera, 2012), desarrollan la interacción de significados, relaciones y tejidos sociales en un espacio, llevándonos a re-construir el espacio urbano, producto de la complejidad inherente a este fenómeno, el cual, no puede ser comprendido sin proponer una interacción entre los planos señalados anteriormente.

De esta manera, la ciudad de Chiguayante puede ser comprendida desde esta lógica, otorgando una nueva visión sobre el plano urbano al vincular elementos sociales y físicos, que inicialmente, no han sido tomados en cuenta en la construcción de la ciudad.

Chiguayante, desde un acercamiento presentado por Vera (2012), implica desarrollar una relación entre las características de la morfología urbana y sus efectosrelaciones con la vida social. Así, se levanta la categoría de "ciudad dormitorio-pendular", que se construye producto la función, el plano, estratos socioeconómicos, conectividad e interacción económica con la conurbación del Gran Concepción.

Los informantes claves, presentan elementos que levantan a la ciudad dormitorio, como un imaginario, producto de que sus funciones laborales, académicas y recreativas son realizadas en otra ciudad-espacio. Potenciando, una idea colectiva de que la ciudad, presenta mayoritariamente, una función de descanso familiarprivado, pero no una función de interacción colectiva. Este último elemento, esta incidido por el plano de la ciudad y la trama urbana que segmenta la ciudad, disminuyendo la posibilidad de una interacción entre los sujetos.

En conclusión, la morfología urbana de Chiguayante configura las relaciones privadas y públicas de los residentes, pero entendiendo, que la urbanitas, interactúa con la conurbación del Gran Concepción. Por lo tanto, lo planteado por Rizo (2006) y Zusman (2013) tiene una alta congruencia, ya que las categorías de lugar y territorio se construyen por la interacción con un espacio físico, implicando una experiencia espacial.

\section{Espacio social}

Esta temática desarrolla 3 dimensiones: uso del espacio, las organizaciones sociales y la oferta de servicios. La primera dimensión, presenta la identificación de lugares reconocidos, los espacios de conmemoración y la vida social en los EP. Luego, la dimensión de organizaciones sociales, describe la participación, el desconocimiento, las acciones y limitantes de participación en las organizaciones sociales. Por último, la dimensión oferta de servicios presenta códigos relacionados a la capacidad de oferta presentes en la ciudad de Chiguayante y Concepción.

La dimensión de uso del espacio presenta los códigos de:

Lugares reconocidos. Existe un reconocimiento de lugares en la ciudad de Chiguayante, pero la escasa interacción social en los lugares de la ciudad inhibe el desarrollo de un tejido social fuerte.

La calle central...creo que por los desfiles es importante, además se tiran los fuegos artificiales y vienen locos a tocar. También es porque como que puede quedar en el centro, entonces van de todos lados. 
Creo que Chiguayante igual es reconocido por Leonera, todos saben dónde queda y además siempre ocurren cosas.

Otro como nuevo, podrían ser las canchas de 8 Oriente, creo que es porque acá no hay club de futbol y además viene harta gente de Conce a jugar (Entrevistado 8).

Los espacios de conmemoración. Son reconocidos dos lugares, la Av. los Héroes y el sector Los Castaños, estando relacionados a festividades, como año nuevo y 18 de septiembre, pero que, en la continuidad del año escolar, no presentan una mayor significancia para los residentes.

Antes como que no había un espacio, pero siento que este último tiempo, es como el espacio que incluso es como un emblema, ahí se hacen todas las actividades (sector Los Castaños) (Entrevistado 8).

La vida social. La interacción social de los residentes de la ciudad, está enfocada principalmente en su plaza principal, ya que, es la única que presenta características de mayor amplitud, pero su accesibilidad y conexión presentan serias dificultades, producto de una vía de alto uso y una vía férrea que conecta la ciudad con la conurbación urbana del Gran Concepción.

La dimensión de organizaciones describe:

La participación. Se otorga una importancia hacia la existencia de las organizaciones, pero no necesariamente su participación en ellas, otorgando la responsabilidad de sus acciones a terceros.

El desconocimiento. No existe un conocimiento sobre la cantidad o el tipo de organizaciones sociales en la ciudad, describiendo una relación con instituciones deportivas o con la municipalidad.

No conozco (organizaciones sociales de Chiguayante), ahí sí que no tengo idea. No cacho nada de eso en verdad, así que no me meto ahí (Entrevistado 14).

Sus acciones. Se otorga acciones importantes como el cierre de la cantera, sector Lonco, contactos para construcción de lomos de toro, obras pequeñas, actividades deportivas y talleres en barrios.

Recuerdo una, que es muy importante, por lo menos para nosotros, que fue el cierre de la cantera... aquí se movieron los vecinos a través de la junta y lograron algo muy importante (Entrevistado 3).

Los limitantes-intereses. El escaso interés por las organizaciones, el modo de vida vinculado a lo laboral, el desconocimiento de su existencia y su funcionamiento

Por mi trabajo no puedo y no tengo idea de que pasa en Chiguayante, uno cuando es comerciante se esclaviza mucho y no puede tener más responsabilidades (Entrevistado 10).

La dimensión de oferta de servicio conlleva los códigos de:

Capacidad de oferta para Concepción. Otras ciudades de la conurbación del Gran Concepción, ofrecen a los residentes de Chiguayante trabajo, estudio, recreación, lugares de entrenamiento.

Si, obvio. Creo que la razón principal es que la mayoría estudia o trabaja allá, entonces pasan la mayoría del tiempo allá (Entrevistado 7).

Capacidad de oferta para Chiguayante. La ciudad, se presenta como ciudad dormitorio, con un escaso tejido social, construyendo un imaginario urbano de "ciudad dormitorio".

La topofilia desde la epistemología de la geografía, en un sentido ontológico, presenta tres categorías de análisis espacial, lugar, hábitat y territorio. Yory (2006) comprende la topofilia como una actitud política espacializada, en donde, se construyen relaciones entre los sujetos con su entorno.

Desde esta interpretación, podemos comprender que la topofilia de los residentes de Chiguayante, presenta un concepto de entorno muy limitado, específicamente a relaciones privadas, con una escasa interacción con entornos cercanos. Esta realidad, incide directamente en el sentido de pertenencia con la idea de un Chiguayante general, sino que, se reconoce un Chiguayante puntual (Alguacil,2008).

Esta filiación, en el sentido de Yory (2007), construye y apropia el entorno, en función de las características urbanas de la ciudad, que influencia la relación y la percepción sobre los otros sujetos y los otros espacios. 
La emocionalidad en la topofilia (Tuan, 2007; Ortega, 2013) implica que las prácticas espaciales inciden en la emoción-cuerpo. Esta interpretación, al interactuar con nuestra área de estudio, nos permite comprender la experiencia de los urbanitas como una experiencia espacial multi-urbana, en donde, la influencia de Concepción en la construcción de las emociones tiene un fuerte impacto.

Así también, la topofobia se levanta como una teoría que explica, la alta segmentación descrita por los informantes claves, los que evidencian un sentimiento de rechazo o desagrado (Tuan,2011;2013, Pineda, 2013). Los factores que provocan rechazo en los individuos, son los prejuicios en torno a prácticas de sujetos de estratos socioeconómico bajos, la delincuencia, la percepción de seguridad, la luminosidad de los entornos, el uso de los diferentes espacios, la trama urbana, escasez de espacios públicos de calidad, la oferta de servicios de Chiguayante y la relación con la conurbación del Gran Concepción.

Esta orientación hacia el rechazo por parte de los habitantes, denota el origen histórico y el tipo de emplazamiento (Lefebvre,1978; Rodríguez,2008; Mas, 2009), que afecta negativamente en la construcción de las relaciones sociales de los sujetos al determinar el factor económico como la base de la construcción de Chiguayante, potenciando así, una topofobia hacia el extremo más lejano de la ciudad (área IV y V) y una fuerte interacción con Concepción, generando un quiebre del tejido social, económico y funcional que afectan a los urbanitas, disminuyendo la complejidad de la ciudad misma (Dangond, Jolly, Monteoliva y Rojas, 2011).

\section{Conclusiones}

A través de las reflexiones realizadas en esta investigación, sobre los imaginarios urbanos, específicamente sobre topofilia y topofobia, se reconocen diferentes aportes que este enfoque puede presentar tanto a la comprensión de la ciudad y sus dinámicas, las relaciones simbólicas y corporales de los urbanitas con sus lugares y los habitantes de este. En el caso de la ciudad de Chiguayante, se puede apreciar a través de los relatos de sus vecinas y vecinos, como la construcción material de la ciudad tiene una clara influencia hacia los imaginarios de los habitantes, debido a que gran parte de la compresión y significancia arraigada en los lugares relatados por los habitantes tenían una fuerte relación a como se ha construido la ciudad y su influencia hacia el sujetosentimiento.

Chiguayante, debido al contexto económico, geográfico y político del Area Metropolitana de Concepción en el cual se encuentra inserto, ha llevado a una forma particular de construir la ciudad. El plano físico de la ciudad muchas veces presenta una influencia negativa para los vecinos, generando sentimientos topofóbicos. Lo anterior se ve reflejado en reiteradas ocasiones en los relatos vecinales, donde se destacaban como una dispar distribución, descuido, tamaño e ignorancia de los espacios públicos, genera una carga significativa negativa, que por una parte segmenta la utilización de estos y además en casos más extremos expulsa de otros por su carga negativa. Otro punto por destacar en cuanto a la influencia de la morfología urbana tiene que ver como la construcción de una ciudad que tiene como objetivo el servir a otras de mayor actividad económica en un área metropolitana, generan dinámicas urbanas que traen consigo la generación de imaginarios de precariedad, insatisfacción, descuido, peligrosidad, entre otros debido a que las dinámicas diarias (el vivir la ciudad) son realizadas en otros lugares.

La generación de sentimientos topofóbicos en los espacios públicos de Chiguayante, no solo tiene consecuencias de un no uso de los lugares o rechazo a estos, sino también se ve reflejado en la dinámica social de la ciudad. Como se logra apreciar, la ciudad al no presentar lugares que incentiven el uso y, por lo tanto, generadores de encuentro, el tejido social se ve muchas veces disminuido o hasta destruido, generando un circulo vicioso, ya que la inexistencia de interacción entre las y los vecinos fomenta el encuentro en el espacio privado o en otras ciudades.

Por otra parte, la ciudad de Chiguayante presenta algunos espacios cargados de sentimientos topofílicos. Los espacios que más destacados, son las plazas, a pesar de presentar carencias en muchas de estas, además se pone en relevancia la necesidad cuidad y mejorar de mejor manera estos espacios públicos. Entre las que más destacan, son las plazas de la Av. Los Héroes y la del sector Los Castaños, esto debido a las diferentes conmemoraciones que son realizadas en ellas. Según lo anterior planteado, es posible vislumbrar que existen espacios, si bien esporádicos y en lapsos específicos de 
tiempo que son altamente valorados, ya que estos evocan muchas veces a sensaciones de felicidad, agrado, entretención, degustación, entre otras sensaciones que cargan al sujeto-sentimiento de sensaciones positivas y, por lo mismo, estos anclan en el lugar sentimiento topofílicos.

Queda plantearse si a futuro, la ciudad de Chiguayante podrá modificar su imaginario urbano de "ciudad dormitorio-pendular", sustentando un aumento de las interacciones sociales entre las diferentes áreas de la unidad de estudio, equilibrando la relación con el Gran

\section{Referencias}

Alguacil, J. (2008). Espacio público y espacio político. La ciudad como el lugar para las estrategias participativas. Madrid: Universidad Carlos III.

Aliste, E. (2011). Territorio y huellas territoriales: una memoria del espacio vivido en el Gran Concepción, Chile. Desenvolvimento e Meio Ambiente(23), 23-58. https://doi.org/10.5380/dma.v23i0.20911

Aliste, E. (2015). Sustainability and territory: an approach to shape development the perspective of the imaginary. En Werlen, Global Sustainability (pp. 119135). Switzerland: Springer International Publishing.

Aliste, E. (2016). Más allá del espacio geográfico: Henry Lefebvre y las geografías invisibles. Alpha(42), 253$258 . \quad$ https://doi.org/10.4067/s071822012016000100016

Aliste, E., y Nuñez, A. (2015). Las fronteras del discurso geográfico: el tiempo y el espacio en la investigación social. Chungara, 47(2), 287-301. https://doi.org/10.4067/s0717-73562015005000023

Bailly, A. S. (1989). Lo imaginario espacial y la geografía. En defensa de la geografía de las representaciones. Madrid: Anales de Geografía de la Universidad Complutense.

Blanco, J., y Gurevich, R. (2006). Una geografía de las ciudades contemporáneas: nuevas relaciones entre actores y territorio. Buenos Aires: Paidos.

Calvo Martínez, T. (2003). La concepción aristotelica de la amistad. La Rioja: Bitarte.
Concepción, construyendo un imaginario de alta integración por sobre el actual, que presenta una baja integración con matices de segmentación y guiado por lineamientos económico-laborales.

El análisis-reflexivo planteado, espera contribuir al debate del urbanismo, desde una óptica de la geografía urbana-social, otorgando una relevancia a los estudios transdisciplinares, como una vía posible, para el mejoramiento de la planificación y construcción de las ciudades, desde lineamientos horizontales [B]

Dangond, C., Jolly, J., Monteoliva, A., y Rojas, F. (2011). Alguas reflexiones sobre la movilidad urbana en Colombia desde la perspectiva del desarrollo humano. Papel Politico, 16(2), 485-514. Disponible en http://www.scielo.org.co/scielo.php?script=sci arttex t\&pid=S0122-44092011000200007\&lng=en\&tlng=es.

Flick, U. (2004). Introducción a la Investigación Cualitativa. Madrid: Ediciones Morata S. L.

Foulkes, M. (2002). El lugar y el no lugar en la utopía de la ciudad privatizada. Resistencia: Facultad de Arquitectura y Urbanismo, UNNE.

Gallardo Frías, L. (2011). Lugar / No-lugar/ lugar en la arquitectura contemporanea. Madrid: Universidad Politécnica de Madrid.

Garrido, F. (2014). Topofilia, paisaje y sostenibilidad del territorio. Universidad de Jaen: Enrahonar. Quaderns de Filosofía.

Guiñez, D. y Gaete, C. (2013). Diagnóstico de Chiguayante. Concepción: SEREMI de Salud de la Regiòn del Biobío.

Hiernaux, D. (2007). Los imaginarios urbanos: de la teoría y los aterrizajes en los estudios urbanos. EURE, XXXIII(99), 17-30. http://dx.doi.org/10.4067/S025071612007000200003

Kuri Pineda, E. E. (2013). Reprentaciones y sigificados en la relación espacio-sociedad: una reflexión teórica (Vol. 28). Ciudad de Mexico: Sociológia. 
Lefebvre, H. (1978). El derecho a la ciudad. Barcelona: Ediciones Peninsulas.

Lindón, A. (2004). Las huellas de Lefebvre sobre la vida cotidiana. Veredas, 5(8), 41-60. Disponible en https://biblat.unam.mx/es/revista/veredas/articulo/l as-huellas-de-lefebvre-sobre-la-vida-cotidiana

Lindón, A. (2007a). El constructivismo geográfico y las aproximaciones cualitativas. Revista de Geografía del Norte Grande, 37, $05 \quad-21$. https://doi.org/10.4067/s0718-34022007000100001

Lindón, A. (2007b). La ciudad y la ida urbana a través de los imaginarios urbanos. EURE (Santiago), 99(33) http://dx.doi.org/10.4067/S025071612007000200002

Lindón, A. (2009). La construcción socioespacial de la ciudad: el sujeto cuerpo y el sujeto sentimiento. Revista Latinoamericana de Estudios sobre Cuerpos, Emociones y Sociedad, 1. Disponible en http://www.relaces.com.ar/index.php/relaces/article /viewArticle/4

Mas, E. (2009). Ciudad: identidad y rankings. Eure (Santiago), 106(35), 29-49. https://doi.org/10.4067/s0250-71612009000300003

Medel Fernández, M. A. (2016). Interpretación del territorio de Chiguayante a partir de sus vecinos. Topofilia: una herramienta necesaria para superar la crisis urbana. Concepción: Universidad de Concepción.

Municipalidad de Chiguayante. (05 de junio de 2018). Historia de Chiguayante. Chiguayante.cl. Recuperado de

https://www.chiguayante.cl/index.php/informacioncomunal/historia-de-chiguayante

Nogué, J. (2014). Sentido del lugar, paisaje y conflicto. Geopolítica(s), https://doi.org/10.5209/rev geop.2014.v5.n2.48842

Ortega, L., Ley, J., Fimbres, N., y Rojas, R. (2013). Caracterización del Valle Mexicali según sus habitantes. Aproximaciones textuales a la topofilia. Culturales, 2(1). Recuperado de http://www.scielo.org.mx/scielo.php?script=sci artte xt\&pid=S1870-11912013000200002
Pyszczek, O. L. (2012). Los espacios subjetivos del miedo: construcción de la estigmatización espacial en relacion con la inseguridad delictiva urbana. Cuadernos de Geografía: Revista Colombiana de Geografía, 21(1), 41-54.

https://revistas.unal.edu.co/index.php/rcg/article/ view/30694

Rizo, M. (2006). Concepciones para pensar lo urbano. El abordaje de la ciudad desde la identidad, el habitus y las representaciones sociales. Bifurcaciones, 6 . http://www.bifurcaciones.cl/006/bifurcaciones 006 Rizo.pdf

Rojas, C.,Muñiz, I y García, M. (2009). Estructura urbana y policentrismos en el Àrea Metropolitana de Concepción. EURE (Santiago), 106(35). https://doi.org/10.4067/s0250-71612009000200003

Strauss, A., y Corbin, J. (1998). Basics of quialitative research: Techniques and procedures for developing grounded theory. Sage Publications.

Tuan, Y.-F. (2007). Topofilia. Un estudio de las percepciones, actitudes y valores sobre el entorno. España: Melusina.

Tuan, Y.-F. (2011). Topophilia and place experience. Revista digital Geografia, Cultura y Educación(1). Recuperado el 18 de Junio de 2018, de http://revistas.pedagogica.edu.co/index.php/anekum ene/article/view/7077

Tuan, Y.-F. (2013). Space and place. Geograficidade, 4(1), 4-13. Recuperado el 18 de Junio de 2018, de https://dialnet.unirioja.es/servlet/articulo?codigo $=47$ 35039

Uribe, H. (2002). El lugar: entre candados, rejas y miedos. REVISTA BIBLIOGRÁFICA DE GEOGRAFÍA Y CIENCIAS SOCIALES, 7(93). http://www.ub.edu/geocrit/b3w393.htm

Vera, P. (2012). Disputas en las construcción simbólica de las ciudades. El Caso Rosario. La Trama de la Comunicación, 16, 69-86. http://www.scielo.org.ar/scielo.php?script=sci arttex t\&pid=S1668-56282012000100005

Yory, C. (1998). La topofilia: una estrategia para hacer ciudad desde sus habitantes. Recuperado de http://132.248.35.1/cultura/2003/ponencias2/Wpon5.html 
Yory, C. (2003). Topofilia, ciudad y territorio: una estrategia pedagogica de desarrollo urbano participativo con dimensión sustentable para las grandes metropolis de America Latina en el contexto de la globalización: el caso de la ciudad de Bogota (Tesis doctoral). Universidad Complutense de Madrid.

Yory, C. (2006). El concepto topofilia entendido como teoría del lugar. En Lugar y territorio: Una aproximación multidimensional a la noción de espacio habitado para pensar y habitar la ciudad del siglo XXI a partir del concepto de topofilia. Bogotá, Colombia: Universidad https://doi.org/10.2307/j.ctv8j5sf.8

Yory, C. (2007). Del espacio ocupado al lugar habitado: una aproximación al concepto de topofilia. En Lugar y territorio: Una aproximación multidimensional a la noción de espacio habitado para pensar y habitar la ciudad del siglo XXI a partir del concepto de topofilia. Bogotá, Colombia: Universidad Piloto. https://doi.org/10.2307/j.ctv8j5sf.8
Yory, C. (2009a). Hábitat urbano y el derecho a la ciudad: una aproximación desde el concepto de topofilia. Medallín: CEHAO.

Yory, C. (2009b). La construcción social del hábitat. Una aproximación antropo-geográfica a la ciudad latinoamericana, en "clave de derechos", orientada a la recualificación de las políticas públicas en materia de hábitat y calidad de vida. Ciudad de Mexico: CIESAS.

Zusman, P. (2013). La geografía histórica, la imaginación y los imaginarios geográficos. Revista de Geogarfía Norte Grande, 54, $51 \quad$ - 66. https://doi.org/10.4067/s0718-34022013000100004 